\title{
Preference Analysis and Decision Support in Negotiations and Group Decisions
}

\author{
Adiel T. de Almeida ${ }^{1}$. Tomasz Wachowicz ${ }^{2}$
}

Published online: 21 June 2017

(C) The Author(s) 2017. This article is an open access publication

\section{Introduction}

Making individual decisions is often challenging because the decision maker needs to take into consideration many different alternatives and conflicting issues (criteria) at the same time. The situation becomes even more challenging when decisions need to be made jointly or negotiated by many decision makers, each having their own viewpoints, preferences, aspirations or reservation levels. To find decision that would satisfy, at least partially, the needs of all parties involved, quantitative measurements may be introduced that allow the expression of the decision makers' preferences in a more precise (numerical) way. Such measurements facilitate the analysis of how well the different alternatives meet the parties' goals and find those that build an equilibrium from the viewpoint of mutual quality and effectiveness.

Various formal methods and techniques may be applied for supporting group decision makers and the negotiators in defining their goals, eliciting preferences and building the quantitative scoring systems required to evaluate the alternatives. They are mainly derived from the field of the multiple criteria decision making/aiding (MCDM/A; Figuera et al. 2005) and game theory (Brams 2003). However, they need to be modified and adopted so that they fit the context of negotiation and group decision making, and meet the cognitive and perceptional capabilities of all parties involved

Adiel T. de Almeida

almeida@cdsid.org.br

$凶$ Tomasz Wachowicz

tomasz.wachowicz@ue.katowice.pl

1 Center for Decision Systems and Information Development, Universidade Federal de Pernambuco, Recife, Brazil

2 Department of Operations Research, University of Economics in Katowice, Katowice, Poland 
in the decision making process, as required by the principles of negotiation analysis (Raiffa et al. 2002). Recent group decision and negotiation literature provides many examples of research focused on both methodological and organizational issues and referring to the real-world examples or experimental results. For instance, the dedicated context dependent algorithms and protocols for structuring group decision or negotiation problems and processes are developed taking into account various viewpoints represented by all stakeholders involved [e.g. Bichler et al. (2003), Eden (2004)]. New methods and techniques for preference elicitation and scoring systems formation take into account the negotiation process dynamics, imprecise preference information and a changeable nature of the problems [e.g. Guo et al. (2003), Roszkowska and Wachowicz (2015)]. The issues of preference aggregation in a multi-party context are studied in order to find a balance between classic group decision consensual approach and majority or plurality recommendations resulting from various voting procedures [e.g. Kadziński et al. (2013), Morais and Almeida (2012), Nurmi (2002)]. There are also studies devoted to the empirical verification of various decision making approaches, focused on confirming or falsifying the current paradigms and concepts [e.g. Danielson et al. (2008), Vetschera (2007)].

Within this special issue five papers reporting on novel research are presented; each focuses on selected issues of multiple group decision making processes and problem.

\section{Articles in this Issue}

In the first paper Antonio Jimenez-Martin, Eduardo Gallego, Alfonso Mateos and Juan A. Fernandez del Pozo present an extension of the additive multi-attribute utility model with veto values that is used to solve a problem of the restoration of an aquatic ecosystem contaminated by radionuclides. The problem they consider involves partial, imprecise or incomplete information about the alternative performances and decision makers' preferences, which result in a degree of uncertainty. Hence, they implement a dominance measuring method to determine the individual rankings for decision makers and aggregate them into a collective decision taking into account their relative importance. The use of the veto concept in MCDM/A has received attention in recent studies, particularly for its applicability in additive aggregation models, dealing with the compensatory effects of such models, when they are unattractive from the decision makers' preferences point of view.

The second paper is focused on the verification of using surrogate weights in multiple criteria decision making. Mats Danielson and Love Eckenberg examine a problem of determining a reliable system of criteria weights out of the preferential information provided by the decision makers in ordinal way, and verify the performance of selected techniques that operate with surrogate weights. They use different conversions of ordinal defined weights into their cardinal equivalents, among others, the rank sum, rank reciprocal and centroid based weights, and compare their robustness using simulation-based approach. This work explores the use of partial information for preference modeling, which has received a growing attention in literature due to the possibility of reducing elicitation errors when requiring less information from decision makers. 
The third paper considers the multi objective optimization problems. Miłosz Kadziński and Michał K. Tomczyk focus on hybridizing the interactive and evolutionary approach to solving optimization problems assuming that the preference model is obtained from the decision makers in indirect way, i.e. using the preference disaggregation approach. Developing their extensions of NEMO-GROUP method they analyze various ways of determining the compromising solutions with respect to different representative functions derived for individual decision makers or jointly for the whole group of decision makers. They simulate the efficacy of their methods using several problems with different numbers of objectives and decision makers and their pre-defined individual value functions. This way they show that NEMO-GROUP is quite a flexible support tool and that the workload related to the number of pairwise comparisons may be decreased by adjusting the elicitation interval and starting generation of the elicitation.

In the fourth paper Marcella Maia Urtiga, Danielle Costa Morais, Keith W. Hipel and Marc Kilgour analyze the problem of supporting the watershed committees in choosing among combinations of alternatives. They developed a method that allows to rank the combinations of alternatives individually for each decision maker first and then to aggregate the individual results into a group decision applying the voting mechanism. The option prioritizing approach is used to elicit the decision makers' individual preferences during an interactive procedure and by means of ordinal judgements. Then the weighted voting system that implements the classification by quartile is used to find what can be called a fair mutual solution. As an example of using the proposed approach the problem of choosing the alternative to prevent the watershed degradation in Brazil is analyzed.

Acknowledgements We are deeply grateful to our reviewers and advisors for giving their feedback to the authors of our special issue papers. We truly believe that their thorough comments and suggestions played a significant role in improving all the submissions and make our special issue a valuable contribution to group decision and negotiation literature.

Open Access This article is distributed under the terms of the Creative Commons Attribution 4.0 International License (http://creativecommons.org/licenses/by/4.0/), which permits unrestricted use, distribution, and reproduction in any medium, provided you give appropriate credit to the original author(s) and the source, provide a link to the Creative Commons license, and indicate if changes were made.

\section{References}

Bichler M, Kersten G, Strecker S (2003) Towards a structured design of electronic negotiations. Group Decis Negot 12(4):311-335

Brams SJ (2003) Negotiation games: applying game theory to bargaining and arbitration. Routledge, London

Danielson M, Ekenberg L, Ekengren A, Hökby T, Lidén J (2008) Decision process support for participatory democracy. J Multicrit Decis Mak 15(1-2):15-30

Eden C (2004) Analyzing cognitive maps to help structure issues or problems. Eur J Oper Res 159(3):673686

Figuera J, Greco S, Ehrgott M (eds) (2005) Multiple criteria decision analysis: state of the art. Springer Verlag, Boston

Guo Y, Müller JP, Weinhardt C (2003) Learning user preferences for multi-attribute negotiation: an evolutionary approach. In: Proceedings of international central and eastern European Conference on multi-agent systems, Springer, pp 303-313 
Kadziński M, Greco S, Słowiński R (2013) Selection of a representative value function for robust ordinal regression in group decision making. Group Decis Negot 22(3):429-462

Morais DC, de Almeida AT (2012) Group decision making on water resources based on analysis of individual rankings. Omega 40(1):42-52

Nurmi H (2002) Voting procedures under uncertainty. Springer, Berlin

Raiffa H, Richardson J, Metcalfe D (2002) Negotiation analysis: the science and art of collaborative decision making. The Balknap Press of Harvard University Press, Cambridge

Roszkowska E, Wachowicz T (2015) Application of fuzzy TOPSIS to scoring the negotiation offers in ill-structured negotiation problems. Eur J Oper Res 242(5):920-932

Vetschera R (2007) Preference structures and negotiator behavior in electronic negotiations. Decis Support Syst 44(1):135-146 